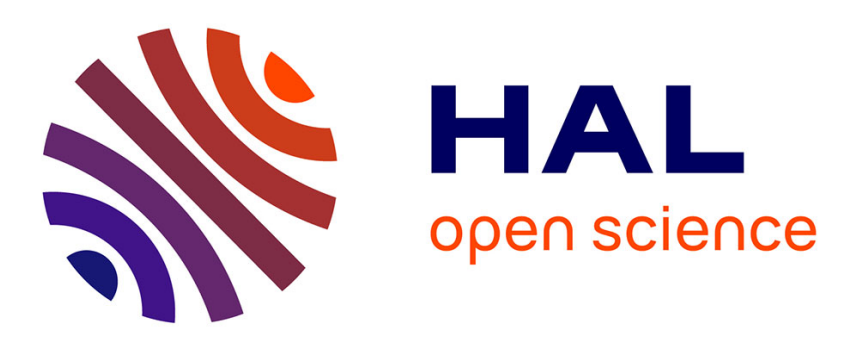

\title{
Statistical Nonlinear Optical Mapping of Localized and Delocalized Plasmonic Modes in Disordered Gold Metasurfaces
}

Gauthier Roubaud, Sébastien Bidault, Sylvain Gigan, Samuel Grésillon

\section{- To cite this version:}

Gauthier Roubaud, Sébastien Bidault, Sylvain Gigan, Samuel Grésillon. Statistical Nonlinear Optical Mapping of Localized and Delocalized Plasmonic Modes in Disordered Gold Metasurfaces. ACS photonics, 2021, pp.1937-1943. 10.1021/acsphotonics.1c00776 . hal-03290440

\section{HAL Id: hal-03290440 \\ https://hal.science/hal-03290440}

Submitted on 19 Jul 2021

HAL is a multi-disciplinary open access archive for the deposit and dissemination of scientific research documents, whether they are published or not. The documents may come from teaching and research institutions in France or abroad, or from public or private research centers.
L'archive ouverte pluridisciplinaire HAL, est destinée au dépôt et à la diffusion de documents scientifiques de niveau recherche, publiés ou non, émanant des établissements d'enseignement et de recherche français ou étrangers, des laboratoires publics ou privés. 


\title{
Statistical Nonlinear Optical Mapping of
}

\section{Localized and Delocalized Plasmonic Modes in} Disordered Gold Metasurfaces

\author{
Gauthier Roubaud, ${ }^{\dagger}$ Sébastien Bidault, ${ }^{*} \uparrow$ Sylvain Gigan,${ }^{\ddagger}$ and Samuel Grésillon ${ }^{*, \dagger}$ \\ $\dagger$ Institut Langevin, ESPCI Paris, Université PSL, Sorbonne Université, CNRS, 1 rue \\ Jussieu, 75005 Paris, France \\ $\ddagger$ Laboratoire Kastler Brossel, Sorbonne Université, École Normale Supérieureiversité PSL, \\ CNRS, Collège de France, 24 rue Lhomond, 75005 Paris, France \\ E-mail: sebastien.bidault@espci.fr; samuel.gresillon@espci.fr
}

\begin{abstract}
Using a statistical analysis of nonlinear luminescence images measured with randomly wavefront-shaped femtosecond excitations, we provide direct insight on both the localized and delocalized plasmonic modes featured by disordered gold metasurfaces. We can image independently areas where far-field wavefront shaping can control the optical properties, and areas with strong subwavelength optical hotspots. In practice, the fraction of the disordered plasmonic surface on which wavefront control is feasible depends strongly on the nanoscale morphology of the sample. Close to the percolation threshold, the entire surface is sensitive to wavefront shaping and we observe the largest densities of delocalized modes as well as the strongest optical hotspots. These results demonstrate how statistical imaging schemes can offset the complexity of disordered nanophotonic systems in order to characterize their optical properties.
\end{abstract}


Keywords: disordered media, plasmonics, metasurfaces, nonlinear luminescence, wavefront shaping

The complex interplay between localized and delocalized plasmonic modes provides disordered metallic metasurfaces with remarkable optical properties. ${ }^{1-3}$ Not only do they feature deeply subwavelength optical field enhancements ${ }^{4-7}$ - or hotspots - with applications in surface enhanced Raman scattering, ${ }^{8}$ nonlinear optics ${ }^{9-13}$ or Purcell effects; ${ }^{14-17}$ but their coupled plasmonic modes, delocalized over micrometer scales, ${ }^{2,3,18}$ permit a far-field optical modulation of the optical response by wavefront shaping. ${ }^{18-22}$ In particular, we recently demonstrated that this combination of near- and far-field phenomena allows the nonlinear photoluminescence (NPL) of gold metasurfaces to be locally increased by more than 2 orders of magnitude, using a femtosecond excitation with a phase profile optimized by an iterative random algorithm. ${ }^{22}$

These optical properties are strongly sensitive to the level of structural disorder of the samples: the strength and density of optical hotspots, ${ }^{6}$ the observed Purcell factors ${ }^{15}$ and the length of the delocalized modes ${ }^{18}$ all depend on the filling fraction $f f$ of gold on the metasurface. In practice, all processes are maximized when the surface is close to the electrical percolation threshold and features the highest morphological complexity, leading to the most efficient wavefront control of nonlinear optical properties. ${ }^{22}$ However, it is currently difficult to associate the deeply subwavelength dimensions of optical hotspots ${ }^{4}$ and the micrometer scales of delocalized modes ${ }^{18}$ in full-field electrodynamic calculations ${ }^{12}$ in order to analyze the optical properties of disordered plasmonic surfaces. Getting an experimental insight into the spatial distributions of localized and delocalized modes is therefore essential to fully exploit such disordered nanophotonic systems.

The analysis of optical hotspots typically requires subwavelength imaging techniques such as near-field optical microscopy, ${ }^{4,5}$ photoemission electron microscopy (PEEM) ${ }^{6,21}$ or spatially resolved electron energy loss spectroscopy (EELS). ${ }^{7}$ However, far-field techniques such as 
$\mathrm{NPL}^{9}$ or second harmonic generation imaging ${ }^{10}$ are also strongly sensitive to the spatial distribution of local field enhancements. Interestingly, it is possible to observe a direct correlation between NPL images and the spatial distribution of optical near-fields ${ }^{23}$ as long as the number of optical hotspots per diffraction limited area is below 1. This low hotspot density is typically observed in disordered gold metasurfaces as experimentally evidenced in PEEM. ${ }^{6}$

On the other hand, an accurate mapping of disordered plasmonic modes remains an open issue. For instance, images obtained by measuring widefield scattering or nonlinear photoluminescence combine intertwined information on both localized and delocalized modes. The intensity measured in a given diffraction-limited area is due to both the strength of local optical hotspots and constructive or destructive interference effects between propagating plasmonic modes. ${ }^{18,22}$ Therefore, we propose here a novel imaging approach that counterbalances this complexity by a statistical analysis of a large number of widefield NPL images obtained with random wavefronts. In particular, we demonstrate that average intensities provide an image of localized plasmonic hotspots with a diffraction-limited resolution; while relative standard deviations yield a map of areas with large densities of interfering delocalized modes, which allow an efficient modulation of nonlinear optical properties by far-field wavefront shaping.

\section{Methods}

Disordered gold metasurfaces are obtained by surface dewetting of a few nanometers of gold deposited on a glass coverslip using an electron-beam (E-Vap Maktek Mighty Source) in a vacuum chamber (Oerlikon). The structural morphology of the surface is characterized in transmission electron microscopy by the filling fraction of gold: $f f=s_{m} /\left(s_{m}+s_{g}\right)$, with $s_{m}$ and $s_{g}$ the surfaces covered by gold and glass, respectively. When the filling fraction reaches a threshold value $f f_{t}=0.65$, electrical percolation is reached. ${ }^{5,24}$ The photoluminescence 

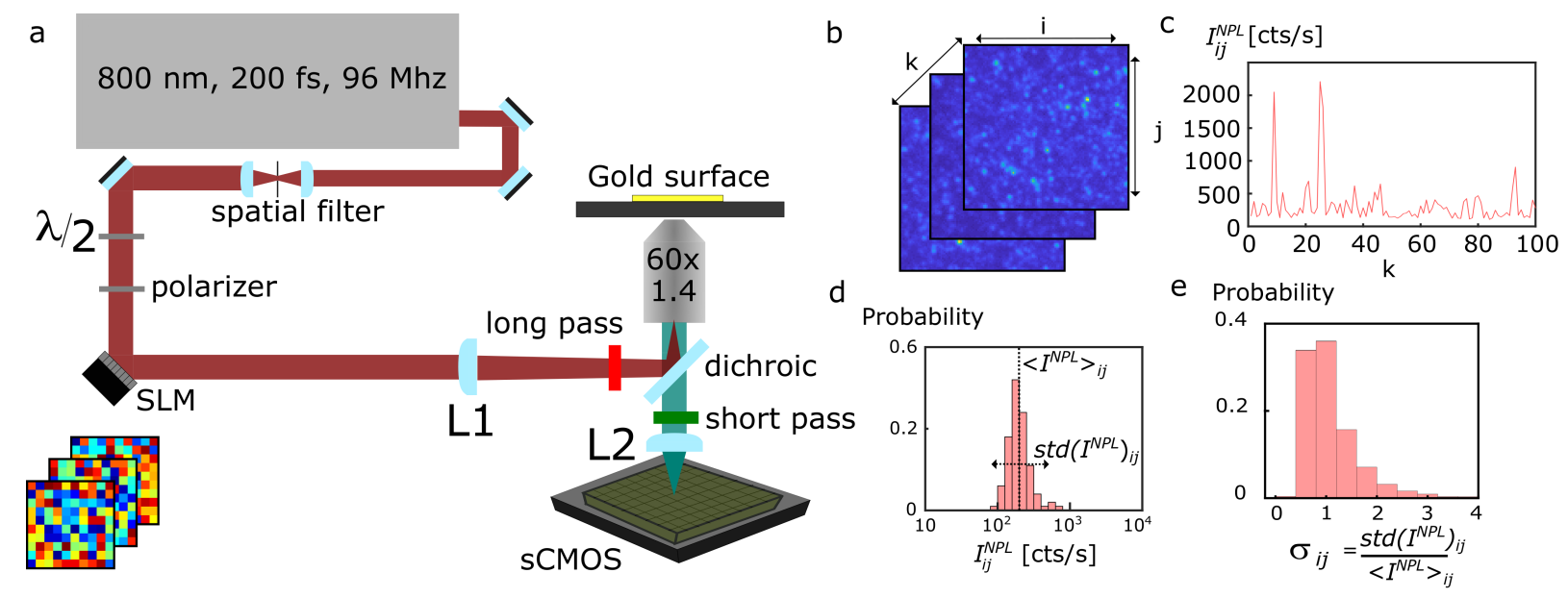

Figure 1: Principle of the experiment. (a) Experimental setup: a spatially filtered and linearly polarized $(\lambda / 2$ : half-wave plate) femtosecond laser is reflected by a spatial light modulator (SLM) before being conjugated to the sample plane and focused on the Fourier plane of the microscope by L1. Nonlinear luminescence (NPL) is focused on a sCMOS camera by L2. A set of spectral filters and a dichroic mirror are used to minimize stray laser light on the camera. (b) NPL images are measured for 100 different random phase patterns on the SLM. (c) Typical NPL intensities for a given $(i, j)$ pixel of the camera when changing the wavefront 100 times. (d) Distribution of NPL intensities for a given $(i, j)$ pixel, allowing the definition of the average intensity, $\left\langle I^{N P L}\right\rangle_{i j}$, and the corresponding standard deviation, $\operatorname{std}\left(I^{N P L}\right)_{i j}$. (e) Distribution of normalized standard deviations $\sigma_{i j}=$ $\operatorname{std}\left(I^{N P L}\right)_{i j} /<I^{N P L}>_{i j}$ for $10^{4}$ pixels of the camera.

of disordered metasurfaces with different gold filling fractions is imaged in parallel using a wavefront-shaped femtosecond excitation as shown on Fig. 1.

As shown in Fig. 1-a, a collimated femtosecond laser (Mira 900, Coherent, 200 fs, 80 MHz, 790 $\mathrm{nm}$ ) is reflected by a spatial light modulator (SLM, LCOS 10468-02, Hamamatsu, 792×600 pixels, $20 \mu \mathrm{m}$ pixel size) before being conjugated by a lens L1 with the gold metasurface using a 1.4 NA $(60 \times)$ objective in an inverted microscope (Eclipse Ti, Nikon). To ensure that the excitation intensity is homogeneous over the sample, the laser is focused by L1 on the Fourier plane of the objective (typical intensity of $1 \mathrm{~kW} / \mathrm{cm}^{2}, 5$ orders of magnitude below the damage threshold of the metasurface). NPL images are retrieved on a sCMOS camera (Edge 5.5, PCO, $2560 \times 2160$ pixels, $6.5 \mu \mathrm{m}$ pixel size) after spectral filtering between $450 \mathrm{~nm}$ and $650 \mathrm{~nm}$. The interest of using nonlinear photoluminescence compared to linear 
photoluminescence lies in the higher sensitivity of NPL to plasmonic hotspots, ${ }^{9}$ which allows a direct correlation of far-field NPL images to electrodynamic simulations (convoluted by the resolution of the imaging system) when there is less than one hotspot per diffraction-limited area. ${ }^{23}$ Reference measurements are performed on a homogeneous fluorescein sample (1 wt\% in a $1 \mu \mathrm{m}$ thick polyvinyl alcohol film) to analyze experimental fluctuations of the excitation intensity due, in particular, to spherical aberrations experienced by the shaped wavefront when passing through the limited numerical aperture of the objective.

The excitation wavefront is chosen randomly and modified 100 times to measure corresponding NPL images over a 90 seconds period (Fig. 1-a-b). Within these images a $10 \mu \mathrm{m} \times 10 \mu \mathrm{m}$ area is selected, corresponding to $10^{4}$ pixels, in which the excitation intensity is homogeneous when considering a constant wavefront. For each $(i, j)$ pixel, it is possible to measure the NPL intensity fluctuations during the iteration process (Fig. 1-c) and, thus, the intensity distribution when excited by random wavefronts (Fig. 1-d). For each pixel of the image, an average nonlinear photoluminescence intensity $\left\langle I^{N P L}\right\rangle_{i j}$ (using a logarithmic scale for the NPL as shown on Fig. 1-d) and a normalized standard deviation $\sigma_{i j}$ (standard deviation of the distribution $\operatorname{std}\left(I^{N P L}\right)_{i j}$ divided by $\left.<I^{N P L}>_{i j}\right)$ are defined. These measurements clearly evidence the ability of far-field wavefront shaping to modulate the emitted NPL with strong intensity variations, more than 5 times larger than the average signal (see Fig. 1-c).

We use the normalized standard deviation $\sigma_{i j}$ so that the fluctuations of the NPL intensity can be compared between gold metasurfaces with different $f f$ values that typically feature very different luminescence signals. Indeed, as shown on Fig. 2, intensity distributions for the different pixels vary significantly as a function of the gold filling fraction $f f$. To illustrate this effect, we selected two filling fractions: one well below percolation, for which the surface is essentially composed of isolated gold particles with nanoscale spacings ( $f f=0.29$, Fig. 2-a); and one close to percolation that corresponds to a fractal morphology with a combination of complex-shaped gold islands and air nanogaps $(f f=0.58$, Fig. 2-b). For the percolated film, we expect significantly larger NPL intensities as optical hotspots are much stronger. ${ }^{6}$ 

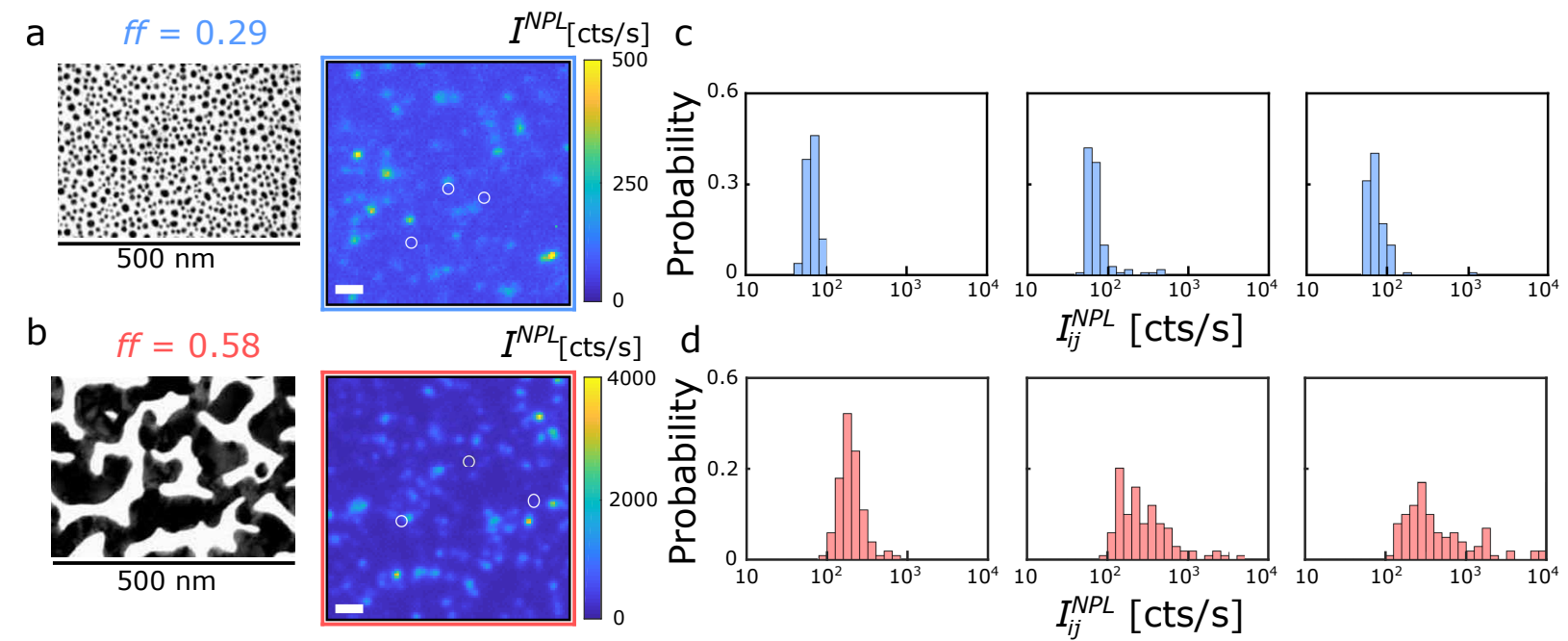

Figure 2: Influence of the gold filling fraction of the disordered metasurface, $f f$, on the measured fluctuations of the nonlinear luminescence (NPL) signal. Typical transmission electron microscopy (left) and NPL (right, bar is $1 \mu \mathrm{m}$ ) images for $f f=0.29$ (a) and $f f=0.58$ (b). Distributions of NPL intensities for three pixels, highlighted by white circles in (a,b), for $f f=0.29$ (blue data, c) and $f f=0.58$ (red data, d).

Furthermore, as shown in Fig. 2-d, the NPL fluctuations are larger with $f f=0.58$, evidencing the more complex nanoscale architecture of the surface observed in the electron microscopy image of Fig. 2-b.

\section{Results and discussion}

Because of the structural complexity of disordered gold metasurfaces, the characterization of their optical properties can only be performed by a statistical analysis. ${ }^{3,6,7,15,17,18}$ For instance, delocalized plasmonic modes were evidenced by analyzing the variance of the scattered optical near-field ${ }^{3}$ and their spatial extent was estimated by studying the variance of the scattered far-field intensity for different illumination conditions. ${ }^{18}$ The influence of localized plasmonic modes can also be observed in the normalized variance of measured Purcell factors. ${ }^{15}$ In general, these statistical analyses are performed over the entire sample by studying different positions on the surface $e^{3,6,7,15,18}$ but our aim here is to provide spatially resolved information. This is why we excite the surface with 100 random wavefronts in order to per- 
form a statistical analysis of the NPL intensity at any given diffraction-limited position of the sample. Compared to previous studies where wavefront-shaping was used to optimize the NPL intensity in a given area of the plasmonic metasurface in a phase-dependent fashion, ${ }^{22}$ this study instead exploits a statistical analysis to infer wavefront-independent information on the sample.

In practice, analyzing the fluctuations of NPL images allows us to map independently areas with strong optical hotspots and areas that feature a large number of interfering delocalized modes. As already mentioned, a widefield nonlinear photoluminescence image mixes the two phenomena. To recover information over the amplitude of optical hotspots, we analyze the average NPL intensity $\left\langle I^{N P L}\right\rangle_{i j}$. Indeed, averaging over 100 excitation conditions cancels out the influence of constructive or destructive interference effects between delocalized plasmonic modes. An image of $\left\langle I^{N P L}\right\rangle_{i j}$ is therefore similar to the image obtained when scanning the surface with a diffraction limited excitation ${ }^{9}$ and provides the relative amplitude of the corresponding excited optical hotspot ${ }^{23}$ as there is typically one hotspot per diffraction-limited area in these gold metasurfaces. ${ }^{6}$

In order to map the local density of delocalized modes on any given point of the sample, it is essential to correct the fluctuations of the NPL signal with respect to its average value. The normalized NPL standard deviation $\sigma_{i j}$ then becomes independent of the strength of local optical hotspots and depends only on interference effects between delocalized plasmonic modes. However, the statistical analysis does not provide quantitative information over the number of localized or delocalized modes. Overall, this statistical imaging scheme recovers, in any diffraction-limited area of the disordered surface, the relative amplitude of optical hotspots and the relative density of interfering delocalized modes.

Apart from interference effects between delocalized modes, several experimental factors can also influence the measured NPL fluctuations. On top of typical experimental noise sources, the sharp phase modulations produced by the SLM introduce small fluctuations of the excitation intensity. Indeed, the magnification of the microscope objective means that the phase 
jumps between SLM pixels are well below the diffraction limit and produce small diffraction fringes. ${ }^{22}$ To characterize these intensity fluctuations convoluted by other measurement noise, we measure two-photon fluorescence (TPF) images of a homogeneous fluorescein-doped film with random phase profiles. These fluctuations are mainly due to the excitation, identical for NPL and TPF, and to the detection path. While there are minute differences in the detection path due to the spectral profiles of NPL and TPF, analyzing the fluctuations of TPF from a homogeneous fluorescein film remains a very good estimate of the excitation fluctuations when measuring NPL from the disordered gold samples. Reference measurements on the gold metasurfaces $(f f=0.29$ and 0.58$)$ and the fluorescein film are also performed using the same incoming wavefront repeated 100 times.

Fig. 3 provides the distributions of normalized standard deviations for the $10^{4}$ pixels of the selected area during these measurements. We observe that distributions of $\sigma_{i j}$ are significantly larger for both metasurfaces under random illuminations compared to all reference measurements, clearly indicating the influence of the sample complexity on the fluctuations of NPL intensities. This is confirmed by the much larger standard deviations for the surface close to the percolation threshold (Fig. 3-b).

Our measurements indicate that the normalized standard deviations observed on the fluorescein film $\sigma_{\text {fluo }}$ (Fig. 3-c and Fig. 3-d) are a good estimator of fluctuations due to both diffraction-induced artefacts of the incoming excitation and to other experimental noise. If the $\sigma_{i j}$ value corresponding to a given pixel in the NPL image of the metasurface is significantly larger than the average $\left\langle\sigma_{\text {fluo }}>\right.$ value for fluorescein, then the NPL fluctuations at this position are due to efficient wavefront shaping of the electromagnetic field distribution on the metasurface and, thus, to strong interference effects between delocalized modes. To estimate with a $98 \%$ fidelity whether a given $(i, j)$ pixel on the NPL images features a large number of interfering delocalized modes, we define a threshold standard deviation value as $\left\langle\sigma_{\text {fluo }}\right\rangle$ plus twice the standard deviation $\operatorname{std}\left(\sigma_{\text {fluo }}\right)$. This threshold is represented in Fig. 3 by a dashed line: it shows that all the pixels, and therefore the entire percolated 

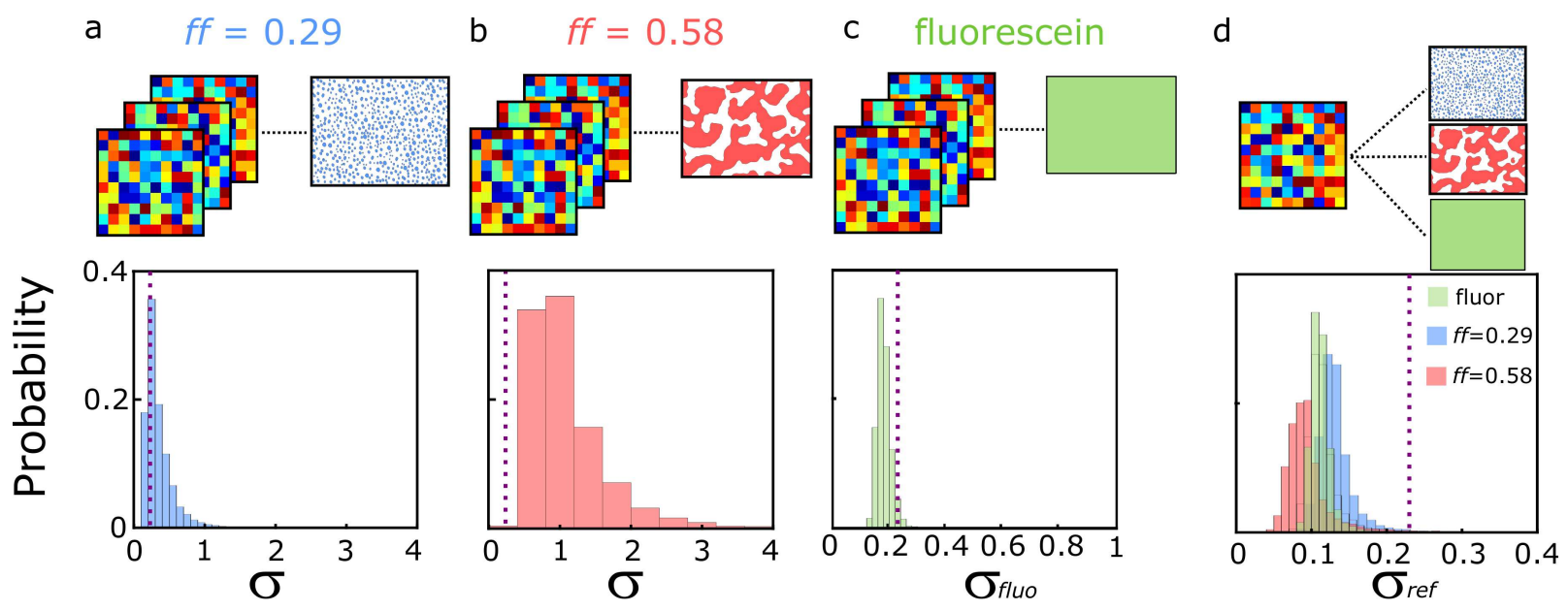

Figure 3: Distributions of normalized standard deviations $\sigma_{i j}$ when exciting gold metasurfaces with $f f=0.29$ (a) and $f f=0.58$ (b) and a homogeneous fluorescein film (c) using 100 different random wavefronts. (d) $\sigma_{i j}$ distributions for the three samples when the same random wavefront is repeated 100 times, used to define our measurement limit. The purple dotted vertical line on all probability distributions corresponds to our measurement limit defined as $<\sigma_{\text {fluo }}>+2 \operatorname{std}\left(\sigma_{\text {fluo }}\right)$.

metasurface $(f f=0.58)$, can be modulated by wavefront shaping, while only a limited part of the low- $f f$ surface will be sensitive to far-field phase fluctuations due to delocalized plasmonic modes. This is not surprising as, for $f f=0.29$, the sample mostly features isolated gold nanoparticles (see Fig. 2-a) for which plasmon polariton propagation is only feasible by plasmon coupling when interparticle gaps are smaller than the particle radii, ${ }^{25}$ a parameter that is not controlled with dewetted gold films. On the other hand, fractal percolated metasurfaces exhibit both elongated particles, on which plasmon polaritons can propagate, and smaller dielectric nanogaps to allow plasmon coupling. This is confirmed by the typical length of delocalized modes, which is more than 2 times larger close to percolation when $f f=0.58$ (more than $4 \mu \mathrm{m}$ ) than for $f f=0.29$ (less than $2 \mu \mathrm{m}$ ), ${ }^{18}$ explaining the typically lower density of such modes for the sample far from percolation.

To more quantitatively characterize the areas of the metasurface that feature a large number of delocalized modes, we propose a relative standard deviation $\Sigma_{i j}$ as a figure of merit of the strength of the NPL fluctuations compared to experimental artefacts as: $\Sigma_{i j}=\sigma_{i j} /\left[<\sigma_{f l u o}>\right.$ 
$\left.+2 \operatorname{std}\left(\sigma_{\text {fluo }}\right)\right]$. Fig. 4 shows the percentage of $\Sigma_{i j}$ values that are larger than 1 as a function of the filling fraction of gold $f f$. A color scheme was designed to highlight the percentage of the surface on which measured fluctuations could arise from experimental artefacts $(0<\Sigma \leq 1)$, compared to areas that are only weakly tunable by wavefront shaping $(1<\Sigma \leq 5)$ and areas with the largest density of delocalized modes $(5<\Sigma \leq 10$ and $10<\Sigma)$.

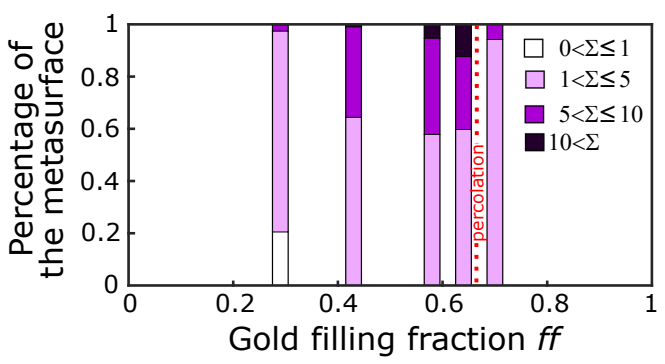

Figure 4: Influence of the gold filling fraction on the percentage of the metasurface that features large values of the fluctuation figure of merit $\Sigma$, ie large fluctuations of the NPL signal with respect to experimental artefacts.

As also demonstrated by the distribution of Fig. 3-a, not all the low- $f f$ sample surface features optical properties that are tunable by wavefront shaping. In practice, about $20 \%$ of the surface does not exhibit a large enough density of delocalized modes $(0<\Sigma \leq 1)$, meaning that NPL fluctuations can be attributed to experimental artefacts. While the rest of the surface is sensitive to far-field phase modifications, the fluctuation figure of merit $\Sigma$ remains below 5 , indicating that a limited number of delocalized modes can effectively interfere to modulate the nonlinear luminescence signal. For all samples close to the percolation threshold or above it, we observe that the figure of merit, $\Sigma$, is larger than 1 for the entire metasurface. This means that far-field wavefront shaping is possible anywhere on samples around or above the percolation threshold. However, we observe that the largest normalized NPL modulations are reached close to the percolation threshold. This is where fluctuations of the nonlinear luminescence can be one order of magnitude larger than those observed on a homogeneous fluorescent layer $(10<\Sigma)$. It is in excellent agreement with previous studies demonstrating longer decay lengths of delocalized modes for metasurfaces close to percolation $^{18}$ or showing that these samples provide the highest NPL enhancement factors after 
wavefront optimization. $^{22}$

The ability of the disordered gold metasurface to feature large local field enhancements, i.e. optical hotspots, and high densities of delocalized plasmonic modes are effectively characterized by the average nonlinear photoluminescence intensity $\left\langle I^{N P L}\right\rangle_{i j}$ and fluctuation figure of merit $\Sigma_{i j}$ for any given position on the sample associated with the $(i, j)$ pixel of the sCMOS camera. Fig. 5-a-d provides typical examples of $10 \mu \mathrm{m} \times 10 \mu \mathrm{m}$ images of $\left\langle I^{N P L}>\right.$ and $\Sigma$ for gold metasurfaces with $f f=0.29$ and $f f=0.58$. We recover the main trends of Fig. 2-4: close to the percolation threshold, both the average nonlinear photoluminescence intensities and the strength of the fluctuations due to wavefront shaping are larger. Since NPL images are diffraction-limited, there is statistically one optical hotspot per measurement area. ${ }^{6}$ This explain why the minimum values of $\left\langle I^{N P L}>\right.$ in Fig. 5-a-b are non-zero. It is also interesting to notice that the largest average NPL intensities in Fig. 5-a-b are significantly lower than the maximum NPL signals in Fig. 2-a-b. As already mentioned, the intensity in widefield NPL images shown in Fig. 2 are due both to local field enhancements and to interference effects between delocalized modes. But in the analysis of Fig. 5 with a large enough number of random wavefronts, the average intensities $\left\langle I^{N P L}\right\rangle$ become independent of interference effects and provide an effective map of the relative intensities of optical hotspots, for a given excitation polarization and wavelength, with diffraction-limited resolution.

Direct comparison between $\left\langle I^{N P L}>\right.$ and $\Sigma$ images do not clearly indicate a spatial correlation between strong optical hotspots (Fig. 5-a-b) and large densities of delocalized modes (Fig. 5-c-d). The calculated 2D correlation coefficients for $f f=0.29$ and $f f=0.58$ are 0.65 and 0.59 , respectively. For instance, the $\left\langle I^{N P L}>\right.$ image in Fig. 5-b indicates optical hotspots with higher amplitudes on the right side of the sample while there are large values of the fluctuation figure of merit $\Sigma$ on the left side of the same area (Fig. 5-d). This demonstrates that the two quantities are not always spatially correlated and highlights the interest of mapping localized and delocalized modes independently.

However, the $50 \%$ of pixels with the largest average NPL intensities have more than a $70 \%$ 


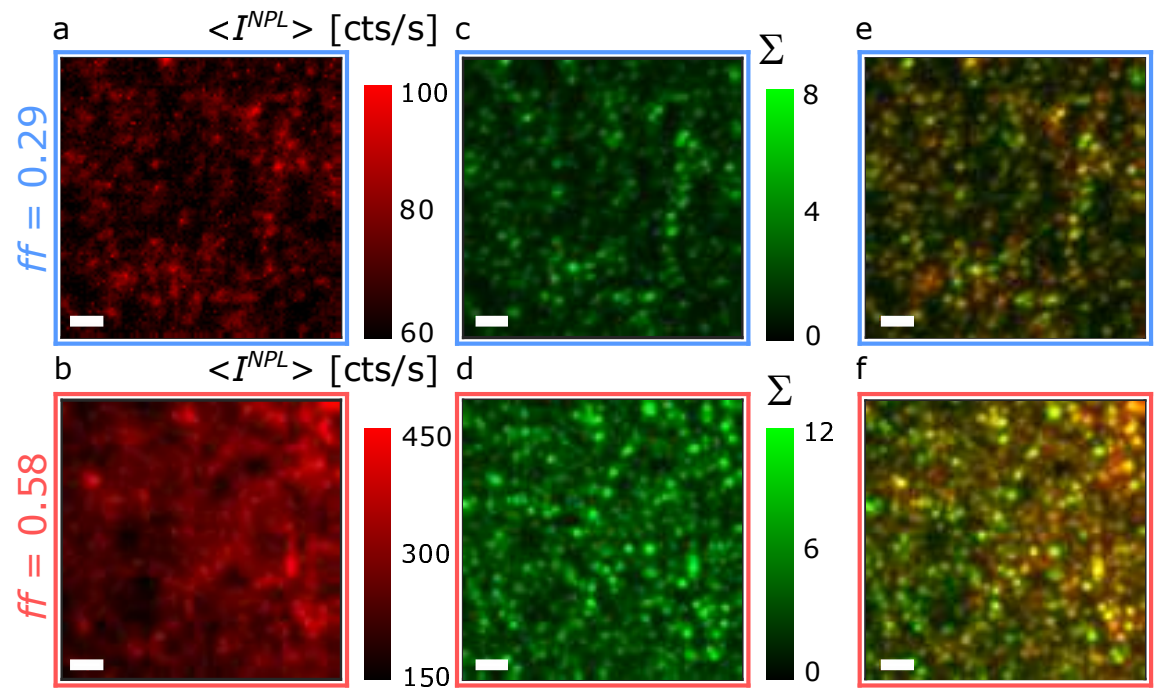

Figure 5: Nonlinear optical mapping of the average NPL intensity $\left\langle I^{N P L}\right\rangle$, associated with local field enhancements $(\mathrm{a}, \mathrm{b})$, of the fluctuation figure of merit $\Sigma$, associated with large densities of interfering delocalized plasmonic modes $(c, d)$ and combined images (e,f) for metasurfaces with a low gold filling fraction, $f f=0.29$ (a,c,e), and one close to percolation, $f f=0.58(\mathrm{~b}, \mathrm{~d}, \mathrm{f})$. Bar is $1 \mu \mathrm{m}$.

chance of exhibiting relative standard deviations above the average value $\langle\Sigma\rangle$. If we now take into account the areas with the largest NPL intensities (first $5 \%$ of $\left\langle I^{N P L}\right\rangle_{i j}$ ), and therefore the highest optical hotspots, the chance of exhibiting relative standard deviations above the average value $\langle\Sigma\rangle$ reach $92 \%$ and $87 \%$ for $f f=0.29$ and $f f=0.58$, respectively. Strong optical hotspots and large densities of interfering delocalized modes are therefore linked. One possible interpretation for this spatial correlation arises from nanoscale air gaps. Indeed, these gaps generate local field enhancements but also favor efficient plasmon coupling for polariton propagation. In practice, the brightest areas of the metasurface have a significant chance of featuring the largest densities of delocalized modes : the $5 \%$ of pixels with highest $\left\langle I^{N P L}\right\rangle_{i j}$ have a $30 \%$ or $65 \%$ chance of having $\Sigma_{i j}$ in the top $5 \%$, for $f f=0.29$ and $f f=0.58$, respectively.

This statistical analysis thus highlights how areas of the disordered gold metasurface can exhibit concurrently strong optical hotspots and large densities of delocalized plasmonic modes. On the other hand, a bright NPL signal does not always ensure efficient wavefront modulation. It is therefore interesting to combine the independent maps of $\left\langle I^{N P L}\right\rangle$ and 
$\Sigma$ as shown on Fig. 5-e,f. Areas that colocalize strong optical hotspots and large densities of delocalized plasmonic modes (which are an essential combination for efficient wavefront shaping) are thus denoted as yellow pixels. On the contrary, mostly red/orange pixels correspond to large average NPL intensities that are weakly sensitive to wavefront shaping, and green pixels indicate large densities of delocalized plasmonic modes with relatively weak optical hotspots.

As discussed above in the statistical analysis of $\left\langle I^{N P L}\right\rangle_{i j}$ and $\Sigma_{i j}$, the areas with the largest average NPL signals generally feature large wavefront modulation. This is especially true for a disordered surface close to the percolation threshold as there is a limited number of bright red pixels in Fig. 5-f. However, it is interesting to note that we observe a number of green-only areas in Fig. 5-e and Fig. 5-f indicating that, for a given excitation polarization and wavelength, parts of the sample that feature interfering delocalized modes will not exhibit the largest NPL intensities, even after wavefront optimization. Statistical imaging can therefore pinpoint areas of the metasurface (denoted here as bright yellow pixels) that are the most likely to provide optimized nonlinear optical properties after wavefront shaping.

\section{Conclusions}

In this report, the fluctuations of nonlinear luminescence produced by disordered gold metasurfaces under a phase-modulated femtosecond excitation were analyzed to provide independent images of areas with large optical hotspots and areas with large densities of interfering delocalized plasmonic modes. The nanoscale morphology of the surface is shown to strongly influence both the average and relative standard deviations of the NPL signal, evidencing the relative strengths of localized and delocalized modes for different filling fractions of gold. In particular, we demonstrate that the amplitude of the localized modes, but also the densities of delocalized modes, are maximized close to the percolation threshold. Importantly, combined images of the average intensities and of the fluctuation figure of merit clearly map 
areas of the metasurface that should provide the highest nonlinear responses after optimizing the phase of the excitation. These results thus demonstrate how statistical imaging schemes can become an essential characterization technique for metasurfaces used in the far-field wavefront control of optical responses, not only to select favourable areas for given excitation conditions, but also to study polarization- and wavelength-dependent memory effects in complex nanophotonic systems.

\section{Acknowledgement}

This work was supported by the French Direction Générale de l'Armement (DGA) under project 2017706. Work at Institut Langevin was supported by LABEX WIFI (Laboratory of Excellence within the French Program "Investments for the Future") under references ANR-10-LABX-24 and ANR-10-IDEX-0001-02 PSL*. Work at Laboratoire Kastler Brossel was supported by the European Research Council (ERC) under project 278025. S.G. is a member of the Institut Universitaire de France (IUF).

\section{References}

(1) Shalaev, V. M., Ed. Optical Properties of Nanostructured Random Media; Springer Berlin Heidelberg, 2002.

(2) Stockman, M. I.; Faleev, S. V.; Bergman, D. J. Localization versus Delocalization of Surface Plasmons in Nanosystems: Can One State Have Both Characteristics? Phys. Rev. Lett. 2001, 87, 167401.

(3) Seal, K.; Genov, D. A.; Sarychev, A. K.; Noh, H.; Shalaev, V. M.; Ying, Z. C.; Zhang, X.; Cao, H. Coexistence of Localized and Delocalized Surface Plasmon Modes in Percolating Metal Films. Phys. Rev. Lett. 2006, 97, 206103. 
(4) Gresillon, S.; Aigouy, L.; Boccara, A. C.; Rivoal, J. C.; Quelin, X.; Desmarest, C.; Gadenne, P.; Shubin, V. A.; Sarychev, A. K.; Shalaev, V. M. Experimental Observation of Localized Optical Excitations in Random Metal-Dielectric Films. Phys. Rev. Lett. 1999, 82, 4520-4523.

(5) Seal, K.; Sarychev, A. K.; Noh, H.; Genov, D. A.; Yamilov, A.; Shalaev, V. M.; Ying, Z. C.; Cao, H. Near-Field Intensity Correlations in Semicontinuous MetalDielectric Films. Phys. Rev. Lett. 2005, 94, 226101.

(6) Awada, C.; Barbillon, G.; Charra, F.; Douillard, L.; Greffet, J.-J. Experimental Study of Hot Spots in Gold/Glass Nanocomposite Films by Photoemission Electron Microscopy. Phys. Rev. B 2012, 85, 045438.

(7) Losquin, A.; Camelio, S.; Rossouw, D.; Besbes, M.; Pailloux, F.; Babonneau, D.; Botton, G. A.; Greffet, J.-J.; Stéphan, O.; Kociak, M. Experimental Evidence of Nanometer-Scale Confinement of Plasmonic Eigenmodes Responsible for Hot Spots in Random Metallic Films. Phys. Rev. B 2013, 88, 115427.

(8) Moskovits, M. Surface Roughness and the Enhanced Intensity of Raman Scattering by Molecules Adsorbed on Metals. J. Chem. Phys. 1978, 69, 4159-4161.

(9) Beversluis, M. R.; Bouhelier, A.; Novotny, L. Continuum Generation from Single Gold Nanostructures through Near-Field Mediated Intraband Transitions. Phys. Rev. $B$ 2003, 68, 115433 .

(10) Anceau, C.; Brasselet, S.; Zyss, J.; Gadenne, P. Local Second-Harmonic Generation Enhancement on Gold Nanostructures Probed by Two-Photon Microscopy. Opt. Lett. 2003, 28, 713-715.

(11) Stockman, M. I.; Bergman, D. J.; Anceau, C.; Brasselet, S.; Zyss, J. Enhanced SecondHarmonic Generation by Metal Surfaces with Nanoscale Roughness: Nanoscale Dephasing, Depolarization, and Correlations. Phys. Rev. Lett. 2004, 92, 057402. 
(12) Chettiar, U. K.; Nyga, P.; Thoreson, M. D.; Kildishev, A. V.; Drachev, V. P.; Shalaev, V. M. FDTD Modeling of Realistic Semicontinuous Metal Films. Appl. Phys. B 2010, 100, 159-168.

(13) Haug, T.; Klemm, P.; Bange, S.; Lupton, J. M. Hot-Electron Intraband Luminescence from Single Hot Spots in Noble-Metal Nanoparticle Films. Phys. Rev. Lett. 2015, 115, 067403.

(14) Biteen, J. S.; Pacifici, D.; Lewis, N. S.; Atwater, H. A. Enhanced Radiative Emission Rate and Quantum Efficiency in Coupled Silicon Nanocrystal-Nanostructured Gold Emitters. Nano Lett. 2005, 5, 1768-1773.

(15) Krachmalnicoff, V.; Castanie, E.; De Wilde, Y.; Carminati, R. Fluctuations of the Local Density of States Probe Localized Surface Plasmons on Disordered Metal Films. Phys. Rev. Lett. 2010, 105, 183901.

(16) Canneson, D.; Mallek-Zouari, I.; Buil, S.; Quélin, X.; Javaux, C.; Mahler, B.; Dubertret, B.; Hermier, J.-P. Strong Purcell Effect Observed in Single Thick-Shell CdSe/CdS Nanocrystals Coupled to Localized Surface Plasmons. Phys. Rev. B 2011, $84,245423$.

(17) Castanie, E.; Krachmalnicoff, V.; Caze, A.; Pierrat, R.; De Wilde, Y.; Carminati, R. Distance Dependence of the Local Density of States in the Near Field of a Disordered Plasmonic Film. Opt. Lett. 2012, 37, 3006-3008.

(18) Bondareff, P.; Volpe, G.; Gigan, S.; Gresillon, S. Probing Extended Modes on Disordered Plasmonic Networks by Wavefront Shaping. ACS Photonics 2015, 2, 1658-1662.

(19) Gunn, J. M.; Ewald, M.; Dantus, M. Polarization and Phase Control of Remote SurfacePlasmon-Mediated Two-Photon-Induced Emission and Waveguiding. Nano Lett. 2006, $6,2804-2809$. 
(20) Gunn, J. M.; High, S. H.; Lozovoy, V. V.; Dantus, M. Measurement and Control of Ultrashort Optical Pulse Propagation in Metal Nanoparticle-Covered Dielectric Surfaces. J. Phys. Chem. C 2010, 114, 12375-12381.

(21) Aeschlimann, M.; Brixner, T.; Cunovic, S.; Fischer, A.; Melchior, P.; Pfeiffer, W.; Rohmer, M.; Schneider, C.; Strüber, C.; Tuchscherer, P.; et al., Nano-Optical Control of Hot-Spot Field Superenhancement on a Corrugated Silver Surface. IEEE J. Sel. Top. Quantum Electron. 2012, 18, 275-282.

(22) Roubaud, G.; Bondareff, P.; Volpe, G.; Gigan, S.; Bidault, S.; Grésillon, S. Far-Field Wavefront Control of Nonlinear Luminescence in Disordered Gold Metasurfaces. Nano Lett. 2020, 20, 3291-3298.

(23) Ghenuche, P.; Cherukulappurath, S.; Taminiau, T. H.; van Hulst, N. F.; Quidant, R. Spectroscopic Mode Mapping of Resonant Plasmon Nanoantennas. Phys. Rev. Lett. 2008, 101, 116805.

(24) Gadenne, P.; Rivoal, J. C. In Optical Properties of Nanostructured Random Media; Shalaev, V. M., Ed.; Springer Berlin Heidelberg, 2002; p 187-215.

(25) Koenderink, A. F.; Polman, A. Complex Response and Polariton-Like Dispersion Splitting in Periodic Metal Nanoparticle Chains. Phys. Rev. B 2006, 74, 033402. 


\section{For Table of Contents Use Only}

Statistical Nonlinear Optical Mapping of Localized and Delocalized Plasmonic Modes in Disordered Gold Metasurfaces

Gauthier Roubaud, Sébastien Bidault, Sylvain Gigan and Samuel Grésillon

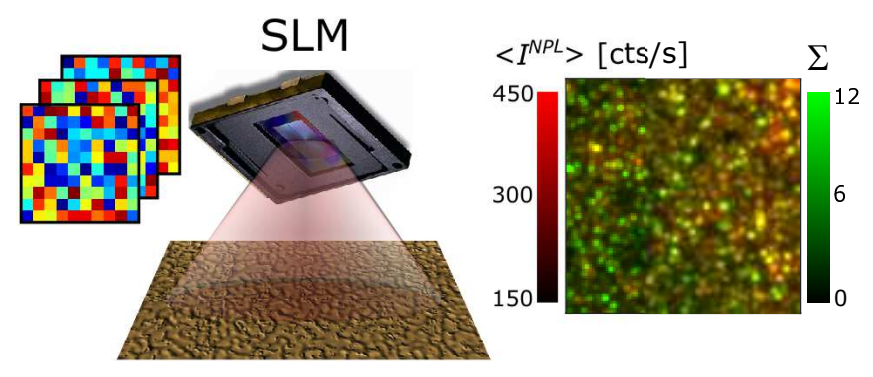

Schematic representation of a disordered gold metasurface excited by random wavefronts generated using a spatial light modulator (SLM, left). Statistical optical map of a percolated gold surface combining an image of the average nonlinear photoluminescence intensity $<I^{N P L}>$, corresponding to localized plasmonic modes, and an image of the fluctuation figure of merit $\Sigma$, corresponding to delocalized plasmonic modes (right). 